\title{
The role of lymphocytes in fetal development and recurrent pregnancy loss
}

\author{
Jan Modzelewski ${ }^{1,}$, Anna Kajdy 1, ${ }^{\text {, }}$, Michal Rabijewski ${ }^{1}$ \\ ${ }^{1}$ Department of Reproductive Health, Centre of Postgraduate Medical Education, Warsaw, Poland \\ ${ }^{2}$ Centrum Medyczne Żelazna, Warsaw, Poland
}

\begin{abstract}
Fetal survival and development is supported by the maternal immune system. Questions regarding those mechanisms have risen from development of transplantation medicine and observation of graft rejection. Initial theories of anatomic division, fetal immune immaturity and maternal immune system inertia were found incorrect. Rejection of fetal "semi-allograft" by maternal immune system could result in pregnancy loss. Two pregnancy losses of any etiology are considered recurrent and effort should be made to name the probable cause. Immune causes of pregnancy loss are probably multifactorial, thus difficult to research and implement findings in clinical practice. Although a full understating of pregnancy loss is not established, new therapies are being developed. This review summarizes the role of lymphocytes in pregnancy development, presents data from studies on recurrent pregnancy loss patients, evidence of new therapies and ESHRE guidelines regarding immunologic investigations.
\end{abstract}

Key words: recurrent pregnancy loss; lymphocytes; Treg; Th17; uNK; abortion; habitual

Ginekologia Polska 2019; 90, 2: 109-113

\section{INTRODUCTION}

From an immunological perspective pregnancy is an interesting phenomenon. On one hand the fetus requires maternal protection from pathogens, on the other develops tolerance for paternal antigens. Interest in immunology of pregnancy started in the beginning of the transplantation era with the observation of transplant rejection mechanisms and natural fetal protection against such rejection.

First, classic theory was presented in 1953 by Peter Medawar, who is considered the founder of reproductive immunology. He described three mechanisms - anatomic division between mother and fetus by placental barrier, antigenic fetal immaturity, and inertia of the maternal immune system [1]. In the following years all three of these mechanisms where questioned and found incorrect [2].

Human placenta stays in contact with maternal blood. Trophoblast cells invade uterine spiral which is a crucial part of placental development. Pathological placentation plays a major role in development of such pregnancy disorders as placenta precerta, acareta, incerta or preeclampsia [3].

Full contact of maternal and fetal cells, especially extravillous trophoblast (EVT) cells, enforce development of an active tolerance to paternal antigens. Fetal antigens are recognized by maternal innate and adaptive immune systems. Both these elements play role in correct development of materno-fetal interface.

\section{KEY LYMPHOCYTES POPULATIONS IN PREGNANCY AND RECURRENT PREGNANCY LOSS}

NK cells are, separate from T and B lymphocytes, lymphocytes with cytotoxic and cytokine producing abilities. Distinct subpopulation of NK called uterine NK ( $\mathrm{UNK}$ ) are present in large numbers in endometrium and decidua [4]. Another lymphocyte populations playing important roles in pregnancy are Treg and Th17 cells. These are two distinct lymphocyte subpopulations of with contradictory roles in the human body. Recent development of reproductive immunology shows that correct balance of these cells may be important in maintaining healthy pregnancy development [5].

Recurrent pregnancy loss (RPL) is defined as loss of two or more pregnancies before reaching viability. Probably $1-2 \%$ of couples suffer from RPL [6]. Most commonly described causes are anatomic defects of the uterus 
(e.g. adhesions, myomas, endometrial polyps and congenital anomalies), chronic endometritis, antiphospholipid syndrome, inherited thrombophilia, endocrine pathologies, fetal and parental genetic factors, and immunological imbalance $[6,7]$. RPL is not only a medical problem of decreased fertility in couples, but also a large psychological burden [8].

At current we can explain only about $50 \%$ of RPL. Probably large proportion of unexplained RPL is caused by immune factors [9]. Research could lead to development of new promising therapies and increase chances for successful pregnancy in couples suffering from RPL.

This paper aims to present a literature review of the role of key lymphocyte populations in RPL, promising therapies of this clinical problem, and available guidelines regarding immunological investigation.

\section{TH17}

Th17 cells are adaptive immunity cells characterized by interleukin-17 (IL-17) production. Other important cytokines produced by Th17 are interleukin-22 (IL-22) and granulocyte-macrophage colony stimulating factor (GM-CSF). Physiologically Th17 cells promote inflammation, especially during bacterial and fungal infection [10].

Th17 cells are formed from naïve $\mathrm{CD} 4^{+} \mathrm{T}$ cells through IL- 6 and TGF- $\beta$ stimulation [11]. These cells possess a certain amount of plasticity that can change cytokine profile to Th1 or Treg. This plasticity is present in vivo during the course of inflammation [12].

\section{TREG}

Treg cells are $\mathrm{CD}^{+}$lymphocytes characterized by expression of forkhead-box P3 (FoxP3) transcription factor. Foxp3 plays a role in immunoregulation. Its deficiency ameliorates the natural history of severe autoimmune disease such as immune dysregulation, polyendocrinopathy, enteropathy, X-linked (IPEX) syndrome [13].

Treg cells regulate immune response by direct interaction with antigen presenting cells, mainly by cytotoxic T-lymphocyte antigen 4 (CTLA-4), secretion of TGF- $\beta$, IL-35 and IL-10 which are anti-inflammatory cytokines, inducing $B$ cell apoptosis through granzymes and high expression of CD25, IL-2 receptor, which depletes IL-2 from the environment [13].

\section{UTERINE NK}

Uterine NK subset is distinct from peripheral NK cells. They have lower cytotoxic and higher immunosuppressive potential. UNK cells are the most abundant lymphocyte subset in human decidua. $60-70 \%$ of all uterine lymphocytes are uNK [14]. Numbers of uNK cells differs during menstrual cycle and pregnancy. In the proliferatory phase uNK compose $10 \%$ of all endometrial stromal cells, $20 \%$ in late secretory phase and even $30 \%$ of stromal cells in the first trimester. Mature forms of human chorionic gonadotropin (hCG) with N-linked carbohydrate side chains act through CD206 (mannose receptor) and enhance uNK proliferation [15].

As other NK cells, uNK are $\mathrm{CD}^{+} 6^{+}$and $\mathrm{CD}^{-}$but lack expression of CD16 which is responsible for antibody dependent cellular cytotoxicity (ADCC). Moreover uNK cells have potential to produce large amounts of cytokines needed in proper development of early pregnancy [15].

Killer immunoglobin-like receptors (KIR) are receptors expressed mainly on NK cells and, depending on type of receptor. KIR binds to trophoblast expressed HLA class $C$ molecules. Certain combinations of KIR and HLA-C haplotypes result in poor uNK activation and increases risk of pregnancy loss. At the same time HLA-G and HLA-E binds to another highly expressed receptor NKG-2A, which result in inhibition of uNK cytotoxicity [15].

\section{IMMUNE SYSTEM INVOLVEMENT IN RPL}

Immunological response to pregnancy probably begins even before pregnancy itself occurs. Murine models show that paternal antigens derived during coitus are detected in female lymph nodes. Two days after coitus, Treg lymphocytes reactive to paternal antigens are present in large numbers in lymph nodes draining the uterus, peripheral lymph nodes and spleen [16]. Interestingly, concentration of TGF $\beta$, cytokine needed in Treg differentiation, in seminal plasma is one of the highest detected in biological fluids [17].

Implantation of conceptus requires a delicate game of pro- and anti-inflammatory factors. IL- 6 which is a potent proinflammatory cytokine and blocker of Treg differentiation [11], also increases trophoblast invasion [3]. Moreover IL- 6 and IL-1 $\beta$ expression is decreased in endometrium of women suffering from pregnancy loss [18] while IL-6 levels in peripheral blood increases $[19,20]$. IL- 6 and IL-1 $\beta$ are pro inflammatory cytokines. Anti-inflammatory cytokine IL-10, which is produced by Treg cells inhibits trophoblast invasion [3].

Further development of fetus is also upkeeped and regulated by the maternal immune system. Decidualization is a process of endometrium remodeling in response to embryo implantation. This results in proper environment for the developing fetus, which needs nourishment from the maternal vascular system. To provide proper blood flow trophoblast cells invade zona intima of maternal spiral arteries and change their morphology allowing increased blood flow. Surprisingly extra villous trophoblast cells form plugs within maternal spiral arteries, until the end of the first trimester. This causes decrease blood flow and enables proper development of the villous trophoblast and arterial remod- 
eling [21]. Process of arterial remodeling is regulated mainly by uNK cells. Immune imbalance is one of the probable etiological factors for idiopathic recurrent pregnancy loss. Such imbalance was detected in several studies. Liu et al. compared lymphocyte numbers from peripheral blood of three groups of patients - unexplained spontaneous recurrent pregnancy loss (URPL), fertile nonpregnant, and pregnant women in confirmed viable pregnancy. Additionally, 6 decidual samples form URPL patients were immunoassayed and compared with samples from elective termination pregnancies. Nonpregnant women and normal early pregnancies had similar number of peripheral Th17 cells. URPL women had significantly higher number of Th17 cells. Number of peripheral Treg cells where similar in nonpregnant and URPL women, while normal early pregnancies had higher number of peripheral Treg cells. Th17/Treg ratio was higher in URPL women than in both other groups. Immunostaining of decidual samples showed higher prevalence of Th17 in decidua of URPL patients than in elective pregnancy termination cases [22].

Lee et al. conducted a study comparing peripheral blood Th17 and Treg ratios in nonpregnant females with URPL and normal fertile women. Study showed statistically higher numbers of Th17 and increased Th17/Treg ratio in URPL women [23].

Study Saifi et al. compared percentage and cytokine profiles of Th17 and Treg lymphocytes in peripheral blood of nonpregnant URPL suffering and fertile women. Fertile women had significantly higher $(9.5 \% \pm 0.52)$ percentage of Treg cells than URPL group $(5.66 \% \pm 0.21)$. Th17 lymphocytes percentage was lower in fertile women $(1.82 \% \pm 0.11)$ than in URPL group $(2.8 \% \pm 0.18)$. Also higher expression of IL- 6 , IL-17 and IL-23 was found in URPL women [20].

Recent study by Qian et al. compared pregnant and non-pregnant URPL women with pregnant and non-pregnant controls. All groups had similar numbers of peripheral blood mononuclear cells but differ in Treg percentage. Pregnant control group had higher proportion of Treg lymphocytes than nonpregnant control, while there was no significant change between pregnant and nonpregnant URPL patients. Moreover, pregnant URPL patients had lower Treg percentage in peripheral blood than pregnant controls. There was no difference in IL-10 and CTLA-4 expression in peripheral blood between groups. They both take action in Treg activation. No difference in Th17/Treg ratio in peripheral blood was found in this study. Investigators collected also decidual samples from URPL patients and women undergoing elective termination of pregnancy. Decreased proportion of Tregs and increased proportion of Th17 was found in URPL patients. Il-10 expression in Treg did not differ between groups, but expression of CTLA-4 was lower in URPL women [5].

\section{POTENTIAL THERAPIES}

With our current knowledge of causes of immune related recurrent miscarriage, arise questions of possible therapies. In fact, there where trials conducted, some with promising results.

Intravenous immunoglobulin (IVIG) administration is proposed to immunomodulate maternal response therefore improving pregnancy outcome. Study of IVIG treatment and NK cell function and levels was conducted by Ahmadi et al. Investigators recruited 78 women with recurrent pregnancy loss, 38 in intervention arm and 40 as a control group. After confirmation of pregnancy, treatment group received $400 \mathrm{mg} / \mathrm{kg}$ IVIG iv. each 4 weeks until 32 weeks of pregnancy. Both groups received standard high-risk pregnancy care. Live birth rate was $86.8 \%$ in treatment group and $45 \%$ in control group $(p=0.0006)$. IVIG treatment also significantly lowered risk of preeclampsia, gestational diabetes and preterm birth. IVIG treatment significantly lowered peripheral NK cell cytotoxicity and frequency. Investigators did not asses uterine NK population [24].

Randomized controlled trials of IVIG treatment yield conflicting results. Most recent metanalysis was performed by Egerup et al. It was conducted according to The Cochrane Handbook for Systematic Reviews of Interventions methodology and strict study protocol. It analyzed outcome of IVIG treatment and scope for calculation of sample size enough to draw definite conclusions. Authors conclude that probably there is a different effect of treatment in primary and secondary recurrent miscarriage patients. Potential beneficial effect of IVIG could be achieved in secondary miscarriage patients, but sample size in both subgroups of metanalysis where too small to establish definite conclusions. Moreover, the treatment group had more maternal adverse effects than placebo group, with no difference in neonatal adverse effects. Authors conclude that there was not enough evidence to give clear clinical recommendations, and IVIG treatment should not be used out of a clinical trial setting. Different conclusions could be made when new evidence will be published [25].

Another widely discussed intervention is paternal or unrelated donor lymphocyte therapy. Most recent metanalysis of this approach was published by Cavalcante et al. It sums up evidence coming from 6 other metanalysis. Four of them found significant improvement in live birth rate, with $O R$ 1.16 (95\% Cl 1.04-1.34), 1.21 (95\& Cl 1.04-1.37), 4.02 (95\% Cl 3.23-5.00), 3.13 (2.56-3.82). One of the metanalyses which shows no improvement with lymphocytes therapy is Cochrane Review published in 2014 [26]. This metanalyses is widely criticized for including one very poor-quality trial showing no effect of lymphocyte therapy. Removal of that study from the Cochrane Review resulted in OR 1.63 (95\% Cl 1.13-2.35) for live birth. In conclusion the authors remarked 
that with improvement of diagnostic and treatment protocols lymphocyte immunotherapy should have its place in RPL treatment [27].

A recently published non-randomized trial by Liu et al. describes successful treatment of 65 patients with low dose $\left(1 \times 10^{7}\right)$ lymphocytes. Investigators showed that this kind of therapy alters unfavorable Th1/Th2/Treg ratio and significantly decreases miscarriage rate from $34.78 \%$ in the control group to $11.68 \%$ in the treatment group. There was no serious adverse events in treatment group, but some patients had reactions in place of administration [28].

Retrospective analysis of 241 patients treated by paternal lymphocyte immunization published by MotakPochrzęst and Malinowski showed promising results. Of 241 patients 206 received 2-6 paternal lymphocytes immunization to induce blocking activity measured by mixed lymphocytes reaction test. The control group were 36 patients with high-risk pregnancy care. Investigators showed increased rate of successful pregnancies in treatment group (83.7\% vs. $36.1 \%, p<0.05$ ) [29].

Retrospective study of Cetin et al. analyzing the use of low molecular weight heparin (LMWH) in selected population of patients with methylene tetrahydrofolate reductase (MTHFR) mutation could be of benefit. 121 women with hetero- and homozygotic MTHFR mutations, 53 in the intervention arm with prophylactic dose of LMWH and 68 in control arm, was included in the study. Both groups received folic acid ( $5 \mathrm{mg} /$ day) and iron ( $80 \mathrm{mg} /$ day) supplementation. LMWH group had higher live birth rate $69.8 \%$ vs. $48.5 \%, p=0.015$ ) and lower congenital anomalies rate (3.3\% vs. $17.6 \%, p=0.022)$. Treatment group delivered two weeks later (34.88 vs. 32.75 ) comparing with supplementation only group but this result did not reach statistical significance $(p=0.060)$ [30].

Recent Bayesian network metanalysis by Lv et al. summed evidence on use of 14 different RPL treatments and placebo. 49 randomized controlled trials and 8469 patients were included. Three different end points - miscarriage, live birth and successful pregnancy defined as birth of a viable fetus, were taken into account. Enough data was available to conclude that treatment with corticosteroids + low dose aspirin + unfractioned heparin, low dose aspirin +, and Granulocyte colony stimulating factor (G-CSF) are effective in decreasing RPL rates and increasing live births in both unexplained RPL and RPL with identified cause. There were no statistically significant differences between each of the mentioned above treatments. Additionally authors made analysis for antiphospholipid syndrome patients and concluded that none of the analyzed treatments performed better than placebo [31].

\section{IMMUNOLOGICAL INVESTIGATIONS GUIDELINES}

The most up to date available guideline is that published by the European Society of Human Reproduction and Embryology. It is a guideline solely dedicated to recurrent pregnancy loss. The guideline was developed based on evidence published until 31 march 2017. In light of current evidence the authors recommend only antiphospholipid syndrome screening, and HLA class II determination in selected population. No other immunological diagnostic tests should be performed, as it won't lead to better treatment or prognosis. Women who fulfil laboratory criteria of phospholipid syndrome and had 3 or more pregnancy losses should be offered low dose aspirin (75-100 mg) before conception and prophylactic dose heparin at time of positive pregnancy test. IVIG ant lymphocyte therapy are not recommended in light of current evidence and should be used only in clinical trial setting. As a result of lack of randomized controlled trials, recommendations are made based on moderate and low quality evidence [6].

\section{SUMMARY}

Reproductive immunology has come a long way since Peter Madawar's first ideas. Yet we are still far from fully understanding exact mechanisms of immunology of embryo development and finding evidence-based treatment for RPL. On the other hand, many trials have brought interesting possibilities and give hope for patients suffering from recurrent pregnancy loss.

Christiansen et al. postulated that because of the evolutionary need for reproductive success most of miscarriages including those of immunological background are multifactorial. A single cause with strong association with recurrent pregnancy loss would be eradicated from gene pool. As a result research and treatment of recurrent pregnancy loss is extremely difficult [9]. Probably there is no universal treatment for women suffering from RPL and combination of therapies tailored for individual patient should be used.

Evidence coming from already conducted trials shows promising results for therapy of immune recurrent pregnancy loss. Most successful therapies seem to be IVIG, paternal lymphocyte therapy and combination corticosteroids + low dose acetylsalicylic acid + unfractioned heparin, GM-CSF, low dose aspirin + low molecular weight heparin.

Although recurrent pregnancy loss research is difficult and full of controversies important progress has been made with hope for finally providing effective care for RPL suffering couples. 


\section{Acknowledgements}

Possible conflict of interest - JM and AK are ESHRE members.

JM participated in all stages of project. AK and MR designed project, participated in data analysis and reviewed final manuscript. AK proofread the manuscript. MR supervised the project.

This project had no external financial support.

\section{REFERENCES}

1. Medawar P. Some immunological and endocrinological problems raised by the evolution of viviparity in vertebrates. Symp Soc Exp Biol. 1953; 7: 320-337.

2. Szekeres-Bartho J. The Role of Progesterone in Feto-Maternal Immunological Cross Talk. Med Princ Pract. 2018; 27(4): 301-307, doi: 10.1159/000491576, indexed in Pubmed: 29949797.

3. Sharma S, Godbole G, Modi D. Decidual Control of Trophoblast Invasion. Am J Reprod Immunol. 2016; 75(3): 341-350, doi: 10.1111/aji.12466, indexed in Pubmed: 26755153.

4. Faas MM, de Vos P. Uterine NK cells and macrophages in pregnancy. Placenta. 2017; 56: 44-52, doi: 10.1016/j.placenta.2017.03.001, indexed in Pubmed: 28284455.

5. Qian J, Zhang Na, Lin J, et al. Distinct pattern of Th17/Treg cells in pregnant women with a history of unexplained recurrent spontaneous abortion. Biosci Trends. 2018; 12(2): 157-167, doi: 10.5582/bst.2018.01012, indexed in Pubmed: 29657243.

6. Atik RB, Christiansen O, Elson J, et al. ESHRE guideline: recurrent pregnancy loss. Human Reproduction Open. 2018; 2018(2), doi: 10.1093/hropen/hoy004.

7. El Hachem H, Crepaux V, May-Panloup P, et al. Recurrent pregnancy loss: current perspectives. Int J Womens Health. 2017; 9: 331-345, doi: 10.2147/IJWH.S100817, indexed in Pubmed: 28553146.

8. Łuczak-Wawrzyniak J, Czarnecka-Iwańczuk M, Bukowska A, et al. Early and late psychological effects of pregnancy loss. Ginekol Pol. 2010; 81(5): 374-377, indexed in Pubmed: 20568519.

9. Christiansen O, Steffensen R, Nielsen $\mathrm{H}$, et al. Multifactorial Etiology of Recurrent Miscarriage and Its Scientific and Clinical Implications. Gynecologic and Obstetric Investigation. 2008; 66(4): 257-267, doi: 10.1159/000149575.

10. Sandquist I, Kolls J. Update on regulation and effector functions of Th17 cells. F1000Res. 2018; 7: 205, doi: 10.12688/f1000research.13020.1, indexed in Pubmed: 29527301.

11. Bettelli E, Carrier Y, Gao W, et al. Reciprocal developmental pathways for the generation of pathogenic effector $\mathrm{TH} 17$ and regulatory T cells. Nature. 2006; 441(7090): 235-238, doi: 10.1038/nature04753, indexed in Pubmed: 16648838.

12. Gagliani N, Amezcua Vesely MC, Iseppon A, et al. Th17 cells transdifferentiate into regulatory $T$ cells during resolution of inflammation. Nature. 2015; 523(7559): 221-225, doi: 10.1038/nature14452, indexed in Pubmed: 25924064.

13. Kawai K, Uchiyama M, Hester J, et al. Regulatory T cells for tolerance. Hum Immunol. 2018; 79(5): 294-303, doi: 10.1016/j.humimm.2017.12.013, indexed in Pubmed: 29288698.

14. Mori M, Bogdan A, Balassa T, et al. The decidua-the maternal bed embracing the embryo-maintains the pregnancy. Semin Immunopathol. 2016; 38(6): 635-649, doi: 10.1007/s00281-016-0574-0, indexed in Pubmed: 27287066.

15. Gong $\mathrm{H}, \mathrm{Chen} \mathrm{Y}, \mathrm{Xu}$ J, et al. The regulation of ovary and conceptus on the uterine natural killer cells during early pregnancy. Reprod Biol Endocrinol. 2017; 15(1): 73, doi: 10.1186/s12958-017-0290-1, indexed in Pubmed: 28874155.
16. Robertson SA, Prins JR, Sharkey DJ, et al. Seminal fluid and the generation of regulatory $T$ cells for embryo implantation. Am J Reprod Immunol. 2013; 69(4): 315-330, doi: 10.1111/aji.12107, indexed in Pubmed: 23480148.

17. Robertson SA, Ingman WV, O'Leary S, et al. Transforming growth factor beta--a mediator of immune deviation in seminal plasma. J Reprod Immunol. 2002; 57(1-2): 109-128, indexed in Pubmed: 12385837.

18. Jasper MJ, Tremellen KP, Robertson SA. Reduced expression of IL- 6 and IL-1alpha mRNAs in secretory phase endometrium of women with recurrent miscarriage. J Reprod Immunol. 2007; 73(1): 74-84, doi: 10.1016/j. jri.2006.06.003, indexed in Pubmed: 17034864.

19. Zhu L, Chen H, Liu M, et al. Treg/Th17 Cell Imbalance and IL-6 Profile in Patients With Unexplained Recurrent Spontaneous Abortion. Reprod Sci. 2017; 24(6): 882-890, doi: 10.1177/1933719116670517, indexed in Pubmed: 27698192.

20. Saifi B, Rezaee SA, Tajik N, et al. Th17 cells and related cytokines in unexplained recurrent spontaneous miscarriage at the implantation window. Reprod Biomed Online. 2014; 29(4): 481-489, doi: 10.1016/j. rbmo.2014.06.008, indexed in Pubmed: 25154016.

21. James JL, Saghian R, Perwick R, et al. Trophoblast plugs: impact on utero-placental haemodynamics and spiral artery remodelling. Hum Reprod. 2018 [Epub ahead of print], doi: 10.1093/humrep/dey225, indexed in Pubmed: 29955830.

22. Liu YS, Wu Li, Tong XH, et al. Study on the relationship between Th17 cells and unexplained recurrent spontaneous abortion. Am J Reprod Immunol. 2011;65(5):503-511, doi: 10.1111/j.1600-0897.2010.00921.x, indexed in Pubmed: 21029245.

23. Lee SK, Kim JY, Hur SE, et al. An imbalance in interleukin-17-producing T and Foxp3 regulatory T cells in women with idiopathic recurrent pregnancy loss. Hum Reprod. 2011; 26(11): 2964-2971, doi: 10.1093/humrep/der301, indexed in Pubmed: 21926059.

24. Ahmadi $M$, Ghaebi $M$, Abdolmohammadi-Vahid $S$, et al. NK cell frequency and cytotoxicity in correlation to pregnancy outcome and response to IVIG therapy among women with recurrent pregnancy loss. J Cell Physiol. 2018 [Epub ahead of print], doi: 10.1002/jcp.27627, indexed in Pubmed: 30317625.

25. Egerup P, Lindschou J, Gluud C, et al. ImmuReM IPD Study Group. The effects of immunotherapy with intravenous immunoglobulins versus no intervention, placebo, or usual care in patients with recurrent miscarriages: a protocol for a systematic review with meta-analyses, trial sequential analyses, and individual patient data meta-analyses of randomised clinical trials. Syst Rev. 2014; 3(10): 89, doi: 10.1186/20464053-3-89, indexed in Pubmed: 25128005.

26. Wong LF, Porter TF, Scott JR. Immunotherapy for recurrent miscarriage. Cochrane Database Syst Rev. 2014(10): CD000112, doi: 10.1002/14651858.CD000112.pub3, indexed in Pubmed: 25331518.

27. Cavalcante MB, Sarno M, Araujo Júnior $E$, et al. Lymphocyte immunotherapy in the treatment of recurrent miscarriage: systematic review and meta-analysis. Arch Gynecol Obstet. 2017; 295(2): 511-518, doi: 10.1007/s00404-016-4270-z, indexed in Pubmed: 28004193.

28. Liu M, Zhen $\mathrm{X}$, Song $\mathrm{H}$, et al. Low-dose lymphocyte immunotherapy rebalances the peripheral blood Th1/Th2/Treg paradigm in patients with unexplained recurrent miscarriage. Reprod Biol Endocrinol. 2017; 15(1): 95, doi: 10.1186/s12958-017-0315-9, indexed in Pubmed: 29246150.

29. Motak-Pochrzest $H$, Malinowski A. Polish experiences with paternal lymphocyte immunization in women with recurrent miscarriages. Neuro Endocrinol Lett. 2015; 36(6): 572-577, indexed in Pubmed: 26812293.

30. Cetin O, Karaman E, Cim N, et al. The impact of low molecular weight heparin on obstetric outcomes among unexplained recurrent miscarriages complicated with methylenetetrahydrofolate reductase gene polymorphism. Ginekol Pol. 2017; 88(5): 260-265, doi: 10.5603/GP.a2017.0049, indexed in Pubmed: 28580572.

31. Lv S, Yu J, Xu X. A comparison of effectiveness among frequent treatments of recurrent spontaneous abortion: A Bayesian network meta-analysis. Am J Reprod Immunol. 2018; 80(1): e12856, doi: 10.1111/aji.12856, indexed in Pubmed: 29709100. 